\title{
The Impact of COVID-19 on the Management of BPH: How Can We Manage the Backlog of Patients?
}

\section{Chughtai $\mathbf{B}^{1}$, Cutone $\mathbf{B}^{2}$, Alshak $\mathbf{M}^{3}$, Mafilios $\mathbf{M}^{4 *}$, Bhattacharyya SK $^{2}$ and Elterman $\mathrm{DS}^{5}$}

${ }^{1}$ Weill Cornell Medical College/New York Presbyterian, USA

${ }^{2}$ Boston Scientific Corporation, Marlborough, USA

${ }^{3}$ Weill Cornell Medical College, USA

${ }^{4}$ Health Economics Associates, USA

${ }^{5}$ University of Toronto, Canada

*Corresponding author: Mike Mafilios, Principal Medical Writer, Health Economics Associates, LLC 1804 Garnet Avenue, Suite 412, San Diego, CA 92109, USA, Tel: 858-367-9140; Email: mafiliosm@hea-consulting.com

\section{Research Article}

Volume 3 Issue 5

Received Date: October 01, 2020

Published Date: October 15, 2020

DOI: $10.23880 /$ jqhe-16000183

\section{Abstract}

It is estimated that more than 30 million elective surgical procedures were cancelled during the initial 12 -week peak of the COVID-19 pandemic, causing an unprecedented backlog of operations in hospitals worldwide. In order to manage this backlog while minimizing in-hospital viral transmission, providers can prioritize elective procedures that use fewer operative services or can be performed outside the hospital setting. We examined physician operative service times for seven surgical techniques for managing benign prostatic hyperplasia and found that minimally invasive surgical techniques take substantially less operative time than more invasive surgical procedures and also offer the option of an entirely out of hospital procedure. As physicians manage the backlog of BPH elective surgeries that were canceled or postponed due to the COVID-19 outbreak, surgical recovery plans should consider BPH procedures that minimize overall operative time in order to reduce the risks of exposing patients to perioperative or iatrogenic COVID-19 infection.

Keywords: Benign Prostatic Hyperplasia; Minimally Invasive Surgical Techniques; Water Vapor Therapy; Surgical Time; Covid-19; Elective Surgery

Abbreviations: COVID-19: Corona Virus; BPH: Benign Prostatic Hyperplasia; PACU: Post Anesthesia Care Unit; TURP: Transurethral Resection of the Prostate; PUL: Prostatic Urethral Lift; AMA: American Medical Association; RUC: RVS Update Committee; TUIP: Transurethral Incision of the Prostate; TUMT: Transurethral Microwave Therapy; HoLAP: Holmium Laser Ablation of the Prostate; HoLEP: Holmium Laser Enucleation of the Prostate; CPT: Current Procedural Terminology.

\section{Introduction}

The 2019 novel corona virus (COVID-19) pandemic has disrupted the delivery of routine hospital services with an estimated 30 million cancellations of operations globally during the initial 12-week peak of the pandemic [1]. Hospitals cancelled elective surgical procedures in order to protect patients from in-hospital viral transmission, free up personal protective equipment, release hospital surgical beds for COVID-19 patient overflow, and redeploy surgical teams to support the COVID-19 public health response [1]. As hospitals resume surgical procedures, time-sensitive operations such as transplant surgeries are expected to be prioritized over conditions such as benign prostatic hyperplasia (BPH) [1]. Moreover, providers will be looking for ways to manage the backlog of procedures that increase operating room 
efficiency, use less surgical time, require minimal time in the post anesthesia care unit (PACU), or that can be performed in alternative care settings such as physician offices. Each year, over 1.2 million surgeries for BPH are performed worldwide [2]. The most common surgical approach is transurethral resection of the prostate (TURP). New minimally invasive surgical techniques (MISTs), such as water vapor therapy or prostatic urethral lift (PUL), may offer a number of advantages including procedural efficiencies and improved morbidity and complication profiles compared to more invasive surgical techniques [3]. Additionally, MISTs offer the option of an entirely in-office (i.e., out of hospital) procedure, further reducing a patient's exposure in the hospital [4]. The present study was undertaken to examine total physician operative service times for seven currently available BPH procedures in the U.S.

\section{Methods}

Physician operative service times were examined for common BPH surgical procedures over a 5-year time horizon (2016 to 2020) using the Medicare Physician Fee Schedule's Physician Time File [5,6]. Data collected for the Physician Time File occurs via the American Medical Association (AMA) RVS Update Committee (RUC) survey administered to appropriate physician specialists nationwide to obtain estimates of the time required to perform a procedure. These estimates are used by Medicare to determine physician reimbursement. The seven BPH procedures examined included TURP, transurethral incision of the prostate (TUIP), transurethral microwave therapy (TUMT), holmium laser ablation of the prostate (HoLAP), holmium laser enucleation of the prostate (HoLEP), PUL, and water vapor therapy. These procedures included a mix of invasive surgical procedures (i.e., TURP, TUIP, TUMT, HoLAP, and HoLEP) and MISTs (i.e., water vapor therapy and PUL). Procedures were identified using Current Procedural Terminology (CPT) codes. The AMA RUC survey defines total physician operative service time as the sum of pre-service time, intra-service time, and post-service time to manage a typical surgical patient [5]. Pre-service time included the evaluation time on the day prior to the procedure as well as the positioning, scrub, and dressing time on the day of the procedure [5]. Intra-service time included the time from the start of the skin incision until the incision was closed ("skin to skin" time) [5]. Post-service time included the time to discharge from recovery [5]. Total physician operative service time did not include the time to diagnose the patient or the post-operative care time after discharge from recovery. PUL times were reported assuming an average of 5 implants (normal range: 4-6 implants) [7]. Water vapor therapy times were based on two-years of the Physician Time File data since the water vapor therapy CPT code was first issued in 2019.

\section{Results}

Median total physician operative services time for each BPH surgical procedure in 2020 varied substantially from 68 minutes for water vapor therapy to 198 minutes for HoLEP procedures (Figure 1).

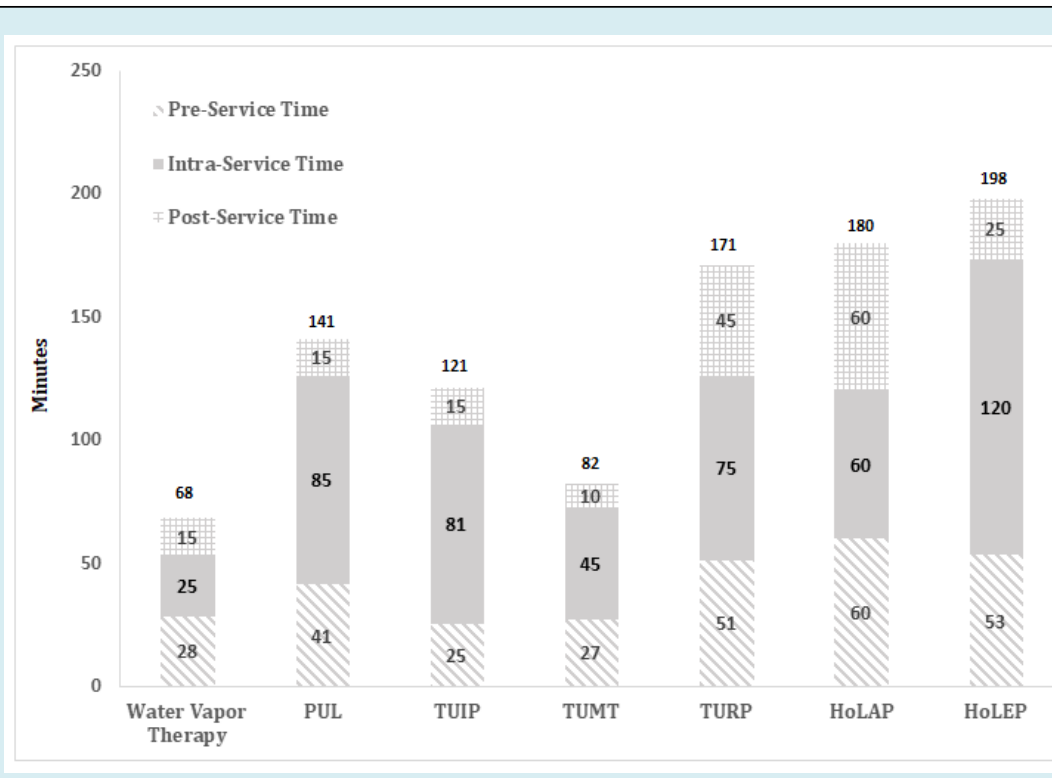

TURP = transurethral resection of the prostate; TUIP = transurethral incision of the prostate; TUMT = transurethral microwave therapy; HoLAP = holmium laser ablation of the prostate; HoLEP = holmium laser enucleation of the prostate; PUL = prostatic urethral lift (5 implants).

Figure 1: Median Physician Operative Services Time (in Minutes) by BPH Surgical Procedure for Year 2020. 
The MISTs (water vapor therapy and PUL) required less total physician operative services time (68 minutes and 141 minutes, respectively) than TURP (171 minutes), the most commonly utilized BPH surgical procedure. The large differences in total physician operative service time between procedures were driven by the intra-service time component. For example, intra-service time accounted for 33\% of total time for water vapor procedures compared to $61 \%$ of total time for HoLEP procedures. Over the 5-year analysis time horizon, total physician operative time decreased by 42 minutes for PUL procedures, decreased by 4 minutes for TURP procedures, and remained the same for all the other procedures.

\section{Discussion}

MISTs for BPH take substantially less operative service time than more invasive surgical procedures. Water vapor therapy in particular requires less operative service time than all of the other procedures. One of the reasons that water vapor therapy and PUL may take less time is that these procedure do not require general anesthesia, and instead, may utilize conscious sedation or a prostate block to manage patient comfort $[7,8]$. This time advantage may allow physicians to handle a larger caseload in a similar amount of time. Additionally, minimally invasive BPH procedures may be advantageous since they can be performed outside the hospital setting in either a physician's office or ambulatory surgical center. As physicians start to manage the backlog of BPH elective surgeries with limited surgical resources, surgical recovery plans should prioritize procedures that minimize operating room time, PACU time, and overall hospital time in order to reduce the risks of exposing patients to perioperative or iatrogenic COVID-19 infection as well as free up operating room resources for other acuity cases delayed by COVID-19. A key strength of this analysis was that the physician operative services time estimates were obtained from the Medicare Physician Time File, a large nationally representative database for surgical procedures performed across many sites and providers based on the AMA RUC survey of physicians. A limitation of this analysis is that the individual components of operative service time may lack precision due to the variance in physician practice patterns in how each procedure is performed across many different sites of care. Consequently, the median physician operative service time may vary substantially from an individual physician's operative experiences.

\section{Conclusions}

The COVID-19 pandemic has caused the cancellation or deferment of many elective BPH surgical procedures. In order to manage the backlog of these procedures, providers should prioritize procedures that minimize operative service times and decrease hospital utilization. MISTs for BPH, specifically the water vapor therapy, take substantially less operative service time and offer the option of an entirely inoffice procedure.

\section{Funding Source}

Support for this research was provided by Boston Scientific Corporation, Marlborough, MA.

\section{Disclosures/Conflict of Interest}

Bilal Chughtai is a board certified urologist at Weill Cornell Medical College and is a paid clinical consultant for Boston Scientific Corporation. Dean Elterman is a board certified urologist at the University of Toronto and is a paid clinical consultant for Boston Scientific Corporation. Samir Bhattacharyya and Ben Cutone are employees of Boston Scientific Corporation. Michael Mafilios is a medical writer employed by Health Economics Associates and was engaged by Boston Scientific Corporation to help develop this manuscript.

\section{References}

1. COVIDSurg Collaborative (2020) Elective surgery cancellations due to the COVID-19 pandemic: global predictive modelling to inform surgical recovery plans. Br J Surg 10.1002/bjs.11746.

2. Riedinger CB, Fantus RJ, Matulewicz RS, Werntz RP, Rodriguez JF, et al. (2019) The impact of surgical duration on complications after transurethral resection of the prostate: an analysis of NSQIP data. Prostate Cancer Prostatic Dis 22(2): 303-308.

3. Christidis D, McGrath S, Perera M, Manning T, Bolton D, et al. (2017) Minimally invasive surgical therapies for benign prostatic hypertrophy: The rise in minimally invasive surgical therapies. Prostate Int 5(2): 41-46.

4. Berardinelli F, Hinh P, Wang R (2009) Minimally invasive surgery in the management of benign prostatic hyperplasia. Minerva Urol Nefrol 61(3): 269-289.

5. Beck DE, Margolin DA (2007) Physician coding and reimbursement. Ochsner J 7(1): 8-15.

6. Hsiao WC, Couch NP, Causino N (1988) Resource-based relative values for invasive procedures performed by eight surgical specialties. JAMA 260(16): 2418-2424.

7. Roehrborn CG, Gange SN, Shore ND (2013) The prostatic urethral lift for the treatment of lower urinary tract symptoms associated with prostate enlargement due to benign prostatic hyperplasia: the L.I.F.T. Study. J Urol 


\section{Journal of Quality in Health care \& Economics}

190(6): 2161-2167.

8. McVary KT, Roehrborn CG (2018) Three-Year Outcomes of the Prospective, Randomized Controlled Rezūm System
Study: Convective Radiofrequency Thermal Therapy for Treatment of Lower Urinary Tract Symptoms Due to Benign Prostatic Hyperplasia. Urology 111: 1-9. 